\title{
A Comparison between Supra-Scarpa's Fascia Abdominoplasty and Traditional Abdominoplasty: Effect of Caesarian Section
}

\author{
Ali Alrida Rahal, Sinan Alboudi, Mhd Husam Alhilbawi, Anwar Alhassanieh \\ Division of Plastic and Reconstructive Surgery, Department of Surgery, Faculty of Medicine, Damascus University, \\ Damascus, Syria \\ Email: Ali.r.raha1988@gmail.com,Sinan986@yahoo.com,h.hilbawi@gmail.com, \\ ragheed.hasan@gmail.com,dranwar_h@yahoo.com
}

How to cite this paper: Rahal, A.A., Alboudi, S., Alhilbawi, M.H. and Alhassanieh, A. (2021) A Comparison between Supra-Scarpa's Fascia Abdominoplasty and Traditional Abdominoplasty: Effect of Caesarian Section. Modern Plastic Surgery, 11, 37-48. https://doi.org/10.4236/mps.2021.112005

Received: April 1, 2021

Accepted: April 27, 2021

Published: April 30, 2021

Copyright $\odot 2021$ by author(s) and Scientific Research Publishing Inc. This work is licensed under the Creative Commons Attribution International License (CC BY 4.0).

http://creativecommons.org/licenses/by/4.0/ (c) (i) Open Access

\begin{abstract}
Background: Abdominoplasty is still among the most popular surgeries in the world of cosmetic surgery, as many modifications have been implemented on this surgery to improve aesthetics and reduce the ratio of complication, especially seroma. Also, the duration to drain removal increases the risk of infection, and this can be avoided by preserving the Scarpa's fascia. Methods: The study was performed from January 2018 to December 2020. The patients were divided into two groups: group A with patients who were performed in Supra-Scarpa's fascia abdominoplasty, and group B who were performed in traditional abdominoplasty; then group A was divided into two subgroups (A1 with caesarean history and A2 with no caesarean history) that differ in caesarean history or not, while a comparison was done between the groups regarding early outcomes. Results: A total of 40 full abdominoplasties were preformed (group A, 19 patients; group B, 21 patients); then group A was divided into two subgroups (group A1, 13 patients; A2, 6 patients); we found that the time until drain removal and its drain output in group $\mathrm{A}$ is less with a statistical significance, and the seroma was less to happen in group A with no statistical significance. However, the operative time in group A is higher with statistical significance. In the subgroups A1 and A2, there was no statistically significant difference in the results. Conclusion: Preservation of the Scarpa's fascia reduces the time until drain removal, total drain output and the occurrence of seroma and the caesarean scar doesn't affect the efficacy of preserving the Scarpa's fascia.
\end{abstract}

\section{Keywords}

Abdominoplasty, Scarpa's Fascia, Caesarian Section 


\section{Introduction}

The anterior abdominal wall is considered one of the most managed areas in the body in the field of cosmetic surgery, and some of the reasons, as known, are sagging after childbirth, diet or bariatric surgery weight change [1]. This body area is one of the most important parts in body harmony, because it is a beauty and function unit, which makes the patient to visit the cosmetic clinic to improve the shape by removing excess skin, repair hernias and diastasis recti if existed, and then improving the physiological state and lifestyle [2] [3].

Despite the good outcomes, the complications ratio remains high. Improvements and modifications have occurred to reduce the ratio of these complications, especially the seroma with a reported incidence from 5\% - 50\% [4] [5] [6] [7]. It has been suggested to perform quilting sutures [8], avoiding electrical cautery [9] and ligating the main vessels to the abdomen [10].

Le Louarn [11] [12] has published, in the 90s of the last century, his technique of preserving the Scarpa's fascia for lowering the seroma rate, as since that time, many studies have confirmed the efficacy of that technique, and some of them have even modified it [13] [14] [15].

The advantages of preserving the Scarpa's fascia are: decreasing the total drain output, decreasing the time to drain removal [16] [17] and contouring the waist [18] [19]. Navois [20] has proved that this method does not influence scar quality and it improves sensibility recovery in the supraumbilical area.

This study aims to compare between the traditional abdominoplasty and preserving a Scarpa's fascia in the early stages, in addition to highlighting the effects of the caesarian section wound on the effectiveness of preserving the superficial fascia.

To our knowledge, a study that mentions the effects of caesarean scar on Supra-Scarpa's fascia abdominoplasty and the final result has not been performed yet.

\section{Patients and Methods}

This study has been initiated on the patients submitted to full abdominoplasty with umbilicus transposition, between January of 2018 and December of 2020, these patients presented with abdominal deformities (excess abdominal skin and adipose tissue with muscle laxity). The ethical confirmation has been acquired from the committee of ethics in the medical college of Damascus university.

The detailed clinical story has mentioned: smoking, Medical comorbidity, history of venus thromboembolism (VTE), focusing on previous abdominal scars and performing a full clinical examination to inspect the existence of rectus abdominis muscles diastasis or hernias. Inclusion criteria: patients presented with anterior abdominal wall deformities including excess abdominal skin and adipose tissue with muscle laxity. Exlusion criteria: patients who were candidates for miniabdominoplasty or circumference or reverse abdominoplasty with the existence of full abdominal liposuction, in addition to the patients without 
weight stabilization for at least 6 months, morbid obesity with BMI more than 40 , or those who anticipate future pregnancy.

The smoking patients have been told to stop smoking for at least 4 weeks before the surgery and 4 weeks others after the surgery, and to stop blood thinner medication 6 days before, and a cardiac counseling has been done for those who have a cardio medical history or who are above 40 years of age.

The patients were recommended to do a full body wash with an antiseptic (Povidone) and clean the umbilicus. The patient is drawn on the traditional known way before the surgery, and is given an intravenous dose of antibiotics prophylacticly.

The surgery is done under general anesthesia, the skin gets cut with a blade reaching the adipose tissue and then the cautery is used with cut settings.

The sample has been divided into two groups: Group A; the dissection is done with a dual-plane on the Scarpa's fascia in the infraumbilical area and above the fascia in the supraumbilical area, in the traditional known way the dissection was in a premuscular plane in the group (B), and reaching the xiphoid in the medial, the dissection is done with extreme caution along the costal margin to preserve the perforators; and the only difference between the two groups is preserving the Scarpa's fascia under the umbilicus while removing a central strip in the medial below the umbilicus to facilitate the xiphopubic fascia plication in group A (Figure $1)$.

Group A has been divided into two groups: group A1; patients with caesarean history and group A2; with no caesarean history.

Fascia plication has done on all patients, with applying an abdominal belt to all of them in the operation room after the surgery, however, liposuction has only been done on the flanks when needed, and a double-pronged closed suction

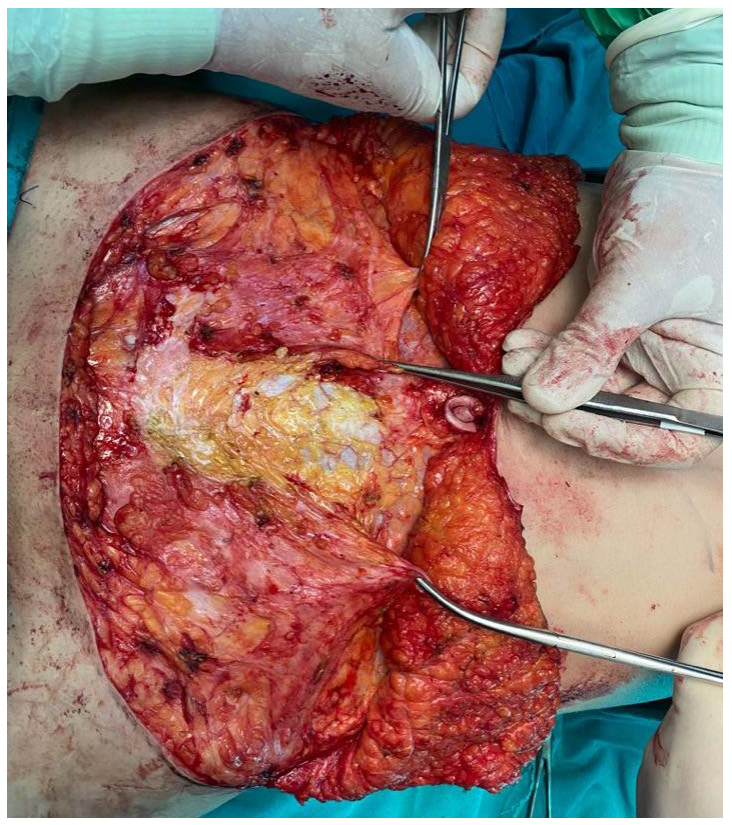

Figure 1. Removing a central strip from the Scarpa's fascia facilitating the plication. 
drain has been placed.

The suture began after folding the operation table, and the patient is discharged the following day, while keeping the high-risk patients in the hospital under observation.

Patients are given these recommendations after the surgery: early ambulation (in a curvy way), sleeping in a curvy way with a slight fold of the knees, keep the abdominal belt worn for 6 weeks while avoid strenuous activity for the same period.

We follow up the patients every day by phone call (at 8 AM) and they are asked about the daily drain output. The drain is removed after 24 hours when the daily collection was less than or equal $25 \mathrm{ml}$. The drain is not removed in the first day for any of the patients.

Check-ups are done weekly after removing the drain for 3 weeks. All of the patients are asked to contact us if anything urgent occurs before the check-up's appointment.

The surgery is done by the senior or the resident with senior observation.

The following variables were recorded: BMI, age, smoking, medication history, previous abdominal surgery, occurrence of seroma, the total drain output, time until drain removal and the operative time.

The seroma was defined as a collection of fluid in the dead space, evident on physical examination after drain removal that was successfully aspirated at least once [16].

Most patients did not make the check-up after the first month.

\section{Statistical Analysis}

The statistical study was carried out using SPSS V.25, the program specialized in analyzing data, studying the differences between values and averages, and testing hypotheses.

\section{Results}

A total of 40 full abdominoplasties were performed in women, 19 of them were performed Supra-Scarpa's fascia abdominoplasty (group A) and the other 21 were performed a traditional abdominoplasty (group B), patients characteristics are summarized in Table 1.

There is no significant statistical difference between the characteristics of both the groups affecting the results.

The previous abdominal operations varied between appendectomy, laparoscopic cholecystectomy and caesarian surgeries (all done in the Pfannenstiel incision).

The results are summarized in Table 2 .

Although group B operative duration was less than group A by only $14 \mathrm{~min}$, it has statistically significant difference $(P<0.05)$, as the average time needed for the surgery in group A was 175.8 minutes, while 161.7 minutes for group $\mathrm{B}$, which opposes to Shahin [21] who has not found a statistical difference in his study. 
Table 1.General characteristics of both groups.

\begin{tabular}{cccc}
\hline & Group A & Group B & P \\
\hline Age & & & \\
Mean \pm SD \\
Range & $39 \pm 8.3$ & $39.5 \pm 9.6$ & NS \\
Bmi & $(27-55)$ & $(22-60)$ & \\
Mean \pm SD & & & \\
Range & & & NS \\
Smoking, total no. (\%) & $29.3 \pm 3.8$ & $28.3 \pm 2.9$ & NS \\
Medical comorbidities, total no. (\%) & $7(63.1-36.6)$ & $(23.8-37.1)$ & NS \\
Previous abdominal surgery, total no. (\%) & $14(73.7 \%)$ & $16(76.2 \%)$ & NS \\
\hline
\end{tabular}

Table 2. Results of both groups.

\begin{tabular}{cccc}
\hline & Group A & Group B & P \\
\hline Operation time & & & \\
Mean \pm SD & $175.8 \pm 21$ & $161.7 \pm 18$ & 0.028 \\
Range & $(120-210)$ & $(120-193)$ & \\
Drain output ( $1^{\text {st }}$ Day) & & & \\
Mean \pm SD & & & 0.013 \\
Range & $102.1 \pm 58.4$ & $145.7 \pm 47.7$ & $(50-270)$ \\
Total Drain output & $(30-250)$ & & \\
Mean \pm SD & & & \\
Range & $206.3 \pm 147.9$ & $390.5 \pm 147$ & $<0.001$ \\
Time until drain removal & $(75-680)$ & $(150-750)$ & \\
Mean \pm SD & & & \\
Range & $3.7 \pm 1.2$ & $5.19+ \pm 1.4$ & 0.002 \\
removal (6 days and more) & $1(5.3 \%)$ & $6(28.6 \%)$ & NS \\
Days until drain reroma, total no. (\%) & $3(15.8 \%)$ & $17(81 \%)$ & $<0.001$ \\
Drain output (300 ml and more) & $0(0 \%)$ & $3(14.3 \%)$ & - \\
\hline
\end{tabular}

The average of drain output was $206.3 \mathrm{ml}$ for group A and $390.5 \mathrm{ml}$ for group B. we found difference with a statistical significance of $(P<0.05)$, which supports Shahin [21] and Ferreira's [16] [22] points.

There was also a statistical difference in the average of time until drain removal. The drains were removed 1.5 days earlier in group A, which approves with earlier studies [16] [21] [23].

And if we got into details, we can see a statistical difference in the average of drain output in day 1 with $43 \mathrm{ml}$ less in group A.

If we calculated the time until drain removal 6 days or more, we found that only one patient (5.3\%) in group A and 6 patients $(28.6 \%)$ in group B with no statistical different $(\mathrm{P}>0.05)$ have overtaken this period, and that result is similar to Ferreira's who found that $32.5 \%$ of the traditional abdominoplasty have 
overtaken the 6 days, in his study, against 1.2\% of them from the supra-Scarpa group [16], in addition to Goncalves [24] with his study for post-bariatric patients. He found that $33 \%$ of the patients had overtaken the 7 days in the traditional group against $1 \%$ in the supra-Scarpa group.

A statistical difference $(\mathrm{P}<0.05)$ has also been found in the drain output that exceeded $300 \mathrm{ml}$ in the both groups, where only 3 patients (15.8\%) had overtaken this amount in group A and 17 patients (81\%) in group B had exceeded the $300 \mathrm{ml}$.

A seroma has been recorded with 3 patients (14.3\%) in group B, while none recorded in group A with no statistical difference $(\mathrm{P}>0.05)$, which approbates with Shahin [21] and his result (15\% for 0\%), and also approves with many studies in terms of reduction the occurrence of seroma. The seroma had been clinically diagnosed and treated with aspiration by needle once for one patient or twice during a week for two.

We divided group A into two subgroups: subgroup A1 with 13 patients with Caesarean section and subgroup A2 with no Caesarean section, with the only variable Caesarean history, as no statistical significance for the differences between the characteristics of these two groups (Table 3).

These was tendency to achieve higher results in group A2 but no statistical difference in the results of : surgeries duration, total draining output, time until drain removal the drain that exceeded $300 \mathrm{ml}$ and Time until drain removal for more than 5 days (Table 4).

\section{Discussion}

This study has revealed that abdominoplasty on a Scarpa's fascia has a clinical effect and a statistical significance in:

- Decreasing the drain output in general.

- Decreasing oozing (less than $300 \mathrm{ml}$ )

- Decreasing the time until drain removal in general.

- A relative increase in the duration of the surgery.

- Decreasing the drain output in the first day.

Table 3. General characteristics of Supra-scarpa Sub-Groups.

\begin{tabular}{cccc}
\hline & Group A1 & Group A2 & P \\
\hline Gender & $13(100 \%)$ & $6(100 \%)$ & - \\
Age & & & \\
Mean \pm SD & $37.46 \pm 8.6$ & $42.33 \pm 7.3$ & NS \\
Range & $(27-55)$ & $(35-53)$ & \\
Bmi & & & \\
Mean \pm SD & & & \\
Range & $29.9 \pm 3.9$ & $27.75 \pm 3.7$ & NS \\
Smoking, total no. (\%) & $(23.8-26.6)$ & $(24.2-32.9)$ & \\
Medical comorbidities, total no. (\%) & $3(23.1 \%)$ & $3(50 \%)$ & NS \\
& $4(30.8 \%)$ & $3(50 \%)$ & NS \\
\hline
\end{tabular}


Table 4.Results of Supra-scarpa Sub-Groups.

\begin{tabular}{cccc}
\hline & Group A1 & Group A2 & P \\
\hline Operation time & & & \\
Mean \pm SD & $174.8 \pm 22$ & $177.8 \pm 20$ & NS \\
Range & $(165-210)$ & $(120-197)$ & \\
Drain output (1st Day) & & & \\
Mean \pm SD & $102.7 \pm 50$ & $100.8 \pm 78$ & NS \\
Range & $(30-200)$ & $(30-250)$ & \\
Total Drain output & & & \\
Mean \pm SD & $198 \pm 103$ & $224.2 \pm 228$ & NS \\
Range & $(80-480)$ & $(75-680)$ & \\
Time until drain removal & & & NS \\
Mean \pm SD & $3.6 \pm 0.7$ & $4 \pm 2.1$ & NS \\
Range & $(3-5)$ & $(2-8)$ & NS \\
\hline Days until drain removal (6 days and more) & $0(0 \%)$ & $1(16.7 \%)$ & $1(16.7 \%)$ \\
Drain output (300 ml and more) & $2(15.4 \%)$ & & \\
\hline
\end{tabular}

It also revealed that abdominoplasty on a Scarpa's fascia has a clinical effect with no statistical significance in:

- Decreasing the long time until drain removal (less than 6 days)

- Decreasing the occurrence of a seroma.

Supra-Scarpa's fascia Abdominoplasty is not a new method. it was suggested by Le Louarn more than 30 years ago with the aim of providing better results and reducing the time needed for recovery, and more importantly to reduce the occurrence of seroma [11] [12] [25].

The fat tissue in the anterior abdominal wall is divided into two parts, superficial and deep, and they are separated by the "Scarpa's fascia" [26] [27]. The superficial layer forms about two thirds of the total abdominal thickness [27] [28].

The lymph drainage, however, happens in two directions. The epigastric area drains to the axillary nodes, while the hypogastric area drains towards the groin nodes [25] [29] [30] [31], while preserving the Scarpa's fascia and the deep fat layer means preserving the lymphatic and blood vessels inside it [17] [25] [32] [33]. The connection is kept between the deep lymphatic vessels-underneath the deep fat layer-to the groin nodes. It also maintains part of blood flow, unlike the second group where the undermining was done directly above the muscular fascia.

The seroma is the most common complications of abdominoplasty, which is defined as the fluids collection (exudate) underneath abdominal flap [34] and it is rich with neutrophils with high percentage of proteins [8], and has been reported to range from 5\% - 50\% [34] [35] [36] [37] [38].

Despite it is normally self-limited, it can accompany secondary complications like wound dehiscence and flap necrosis due to the pressure on the wound and carry the risk of becoming infected, resulting in an abscess. And can lead to the 
development of pseudocyst or pseudobursa, and it needs additional surgical procedures to be removed and may causes deformations on the abdominal wall [6].

Elevation of the skin flap necessarily results in a large elevated surface area that produces serous fluid after the inflammatory response to injury and cuts the lymphatic vessels that drain these fluids, in addition to form an dead space that allows the fluids to be gathered [8] [9] [23].

Preserving the Scarpa's fascia in abdominoplasty can protect some of the physiological structures (the lymphatic and blood vessels) and theoretically leads to reduce bleeding, (maintain the superficial epigastric system) and allow a better adhesion between the abdominal flap and Scarpa's fascia, which hence a less amount of fluids is gathered and reduces the chance of seroma by draining the fluids if gathered through the left deep lymphatic vessels [39]. That is what was confirmed by Friedman [40] when he pointed that the undermining on Supra-Scarpa's fascia in abdominoplasty preserves around $20 \%$ of lymphatic vessels that helps to drain fluids which reduces the drain output.

Le Louarn [11] [12] mentioned that preservation the deep fat compartment respects the anatomic structures of the abdomen and provides a well vascularized tissue with the accompanying lymphatic vessels, which speeds up recovery and reduces seroma occurrence.

Another mechanism has been suggested, explaining the superiority of preserving the Scarpa's fascia, is summarized that the adhesion of the elevated abdominal flap with the "Scarpa's fascia and the deep fat layer underneath" has led to find a good way for providing blood supply, while the existence of a muscular fascia (glistering white surface) in the traditional abdominoplasty with poor blood has played a role in allowing the flap to to glid (shearing movement) [11] [12] [22] [41].

This adhesion, while wearing the abdominal belt, can reduce the existence of the dead space. So both the physiological mechanism (preserving the lymphatic vessels) and the mechanical mechanism (adhesion between the flap and Scarpa's fascia) lead to reduce seroma, and the possibility of the complications resulted by the seroma (clotting, infection and forming a pseudo-bursa).

As we mentioned, preserving a part of the connection between the lymphatic vessels and the groin nodes leads to drain the fluids and helps the suction drain. So the oozing can spread between the suction drain and the lymphatic vessels and reduce the drain output.

The usage of drain has been considered among the precautionary procedures against seroma and hematoma forming [42]. Although it causes discomfort for the patient with the ability of the retrograde bacterial migration through its entrance, especially when kept for a long period [43].

On the other side, reducing the time to drain removal provides a relief to the patient from the discomfort and pain resulted and speeds up her return to her daily life and reduces the possibility of infection that can spread as long as the 
drain is placed.

When comparing the effect of the Caesarean scar to the Scarpa's fascia, we found that the scar has no effect on the efficiency whatsoever.

The caesarean incision (Pfannenstiel incision) crosses all layers in the anterior abdominal wall and cuts the connection between the lymphatic vessels as we mentioned before, and theoretically the sufficiency of Scarpa's fascia should be affected in reducing the total drain output and the time to drain removal and reducing the seroma formation, while practically, this negative impact has not appeared.

There are several analyses: the possibility that most of the lymph vessels are located laterally, therefore preserving its sufficiency or a thought can be given that the deep fat itself observes the oozing or that the impact of the adhesion force which the adhesive surface of fat contains more blood vessels than the ridge surface of the fascia. The most affective impact on the sufficiency of the superficial fascia is reducing the dead space.

Additional studies, for how the lymphatic vessels are located in the lower part of the abdominal wall, must be done or even a study on the impact of these vessels while preserving the Scarpa's fascia to know what the impact of lymphatic vessels and the force of adhesion of each separately.

Unfortunately, some points were not reached for our research due to the small number of people in our sample, which limited our ability to connect the risk factors with complications and the patient did not visit us routinely for check-ups, especially after the first month, to study and follow up with the late complications, and also, the interruption of communication and covid-19 pandemic had a great negative role in the following up. As for the caesarean scar it needs more and wider studying and analyzing.

Multiplicity of surgeon can also have a role in the results, especially the length of the surgical procedure.

\section{Conclusion}

Scarpa's fascia has a positive impact in the time to drain removal and its production and reducing the occurrence of seroma. The caesarean scar doesn't affect the efficacy of preserving the Scarpa's fascia in abdominoplasty procedure.

\section{Conflicts of Interest}

The authors declare no conflicts of interest regarding the publication of this paper.

\section{References}

[1] Rubin, J.P. and Neligan, P.C. (2017) Plastic Surgery E-Book: Volume 2: Aesthetic Surgery. Elsevier Health Sciences, Amsterdam.

[2] Papadopulos, N.A., et al. (2012) Does Abdominoplasty Have a Positive Influence on Quality of Life, Self-Esteem, and Emotional Stability? Plastic and Reconstructive 
Surgery, 129, 957e-962e. https://doi.org/10.1097/PRS.0b013e31824ecc2b

[3] Song, A.Y., et al. (2006) Body Image and Quality of Life in Post Massive Weight Loss Body Contouring Patients. Obesity, 14, 1626-1636.

https://doi.org/10.1038/oby.2006.187

[4] Neaman, K.C., et al. (2013) Outcomes of Traditional Cosmetic Abdominoplasty in a Community Setting: A Retrospective Analysis of 1008 Patients. Plastic and Reconstructive Surgery, 131, 403e-410e. https://doi.org/10.1097/PRS.0b013e31827c6fc3

[5] Costa-Ferreira, A., et al. (2016) Scarpa Fascia Preservation during Abdominoplasty. In: Aesthetic Plastic Surgery of the Abdomen, Springer, Berlin, 59-73.

https://doi.org/10.1007/978-3-319-20004-0 7

[6] di Summa, P.G. and Kalbermatten, D.F. (2016) Abdominoplasty Complications and Seroma: From Prevention to Effective Treatment. In: Aesthetic Plastic Surgery of the Abdomen, Springer, Berlin, 487-491.

https://doi.org/10.1007/978-3-319-20004-0 39

[7] Najera, R.M., et al. (2011) Comparison of Seroma Formation Following Abdominoplasty with or without Liposuction. Plastic and Reconstructive Surgery, 127, 417-422. https://doi.org/10.1097/PRS.0b013e3181f95763

[8] Andrades, P., et al. (2007) Progressive Tension Sutures in the Prevention of Postabdominoplasty Seroma: A Prospective, Randomized, Double-Blind Clinical Trial. Plastic and Reconstructive Surgery, 120, 935-946. https://doi.org/10.1097/01.prs.0000253445.76991.de

[9] Marsh, D.J., et al. (2015) Abdominoplasty and Seroma: A Prospective Randomised Study Comparing Scalpel and Handheld Electrocautery Dissection. Journal of Plastic, Reconstructive \& Aesthetic Surgery, 68, 192-196. https://doi.org/10.1016/j.bjps.2014.10.004

[10] Skillman, J., et al. (2014) Ligating Perforators in Abdominoplasty Reduces the Risk of Seroma. Aesthetic Plastic Surgery, 38, 446-450.

https://doi.org/10.1007/s00266-013-0267-1

[11] Le Louarn, C. (1992) Partial Subfascial Abdominoplasty. Our Technique Apropos of 36 Cases. Annales de chirurgie plastique et esthetique, 37, 547-552.

[12] Le Louarn, C. (1996) Partial Subfascial Abdominoplasty. Aesthetic Plastic Surgery, 20, 123-127. https://doi.org/10.1007/BF02275530

[13] Gardner, P.M. and Vasconez, L.O. (1996) Liposculpture and Lipectomy Superficial to Scarpa's Fascia. Operative Techniques in Plastic and Reconstructive Surgery, 1, 42-46. https://doi.org/10.1016/S1071-0949(96)80050-3

[14] Espinosa-de-los-Monteros, A., et al. (2006) Abdominoplasty with Total Abdominal Liposuction for Patients with Massive Weight Loss. Aesthetic Plastic Surgery, 30, 42-46. https://doi.org/10.1007/s00266-005-0126-9

[15] Saldanha, O.R., et al. (2001) Lipoabdominoplasty without Undermining. Aesthetic Surgery Journal, 21, 518-526. https://doi.org/10.1067/maj.2001.121243

[16] Costa-Ferreira, A., et al. (2013) Scarpa Fascia Preservation during Abdominoplasty: Randomized Clinical Study of Efficacy and Safety. Plastic and Reconstructive Surgery, 131, 644-651. https://doi.org/10.1097/PRS.0b013e31827c704b

[17] Koller, M. and Hintringer, T. (2012) Scarpa Fascia or Rectus Fascia in Abdominoplasty Flap Elevation: A Prospective Clinical Trial. Aesthetic Plastic Surgery, 36, 241-243. https://doi.org/10.1007/s00266-011-9795-8

[18] Mossaad, B.M. and Frame, J.D. (2016) Medial Advancement of Infraumbilical Scarpa's Fascia Improves Waistline Definition in "Brazilian” Abdominoplasty. In: 
Aesthetic Plastic Surgery of the Abdomen, Springer, Berlin, 75-86. https://doi.org/10.1007/978-3-319-20004-0 8

[19] Whiteman, D. and Miotto, G.C. (2016) Abdominoplasty with Scarpa's Fascia Advancement Flap to Enhance the Waistline. Aesthetic Surgery Journal, 36, 852-857. https://doi.org/10.1093/asj/sjv254

[20] Novais, C.S., et al. (2020) Abdominoplasty with Scarpa Fascia Preservation: Randomized Controlled Trial with Assessment of Scar Quality and Cutaneous Sensibility. Plastic and Reconstructive Surgery, 146, 156e-164e. https://doi.org/10.1097/PRS.0000000000007024

[21] Shahin, M.A., Hagag, M.G. and El-Meligy, M.H. (2018) Outcome after Preservation of Scarpa's Fascia in Abdominoplasty. The Egyptian Journal of Surgery, 37, 260. https://doi.org/10.4103/ejs.ejs 1918

[22] Costa-Ferreira, A., et al. (2010) Scarpa Fascia Preservation during Abdominoplasty: A Prospective Study. Plastic and Reconstructive Surgery, 125, 1232-1239. https://doi.org/10.1097/PRS.0b013e3181d0ac59

[23] Fang, R.C., Lin, S.J. and Mustoe, T.A. (2010) Abdominoplasty Flap Elevation in a More Superficial Plane: Decreasing the Need for Drains. Plastic and Reconstructive Surgery, 125, 677-682. https://doi.org/10.1097/PRS.0b013e3181c82f78

[24] Correia-Gonçalves, I., et al. (2017) Abdominoplasty with Scarpa Fascia Preservation-Comparative Study in a Bariatric Population. Surgery for Obesity and Related Diseases, 13, 423-428. https://doi.org/10.1016/j.soard.2016.09.024

[25] Le Louarn, C. and Pascal, J.F. (2000) High Superior Tension Abdominoplasty. Aesthetic Plastic Surgery, 24, 375-381. https://doi.org/10.1007/s002660010061

[26] Markman, B. and Barton Jr., F.E. (1987) Anatomy of the Subcutaneous Tissue of the Trunk and Lower Extremity. Plastic and Reconstructive Surgery, 80, 248-254. https://doi.org/10.1097/00006534-198708000-00015

[27] Costa-Ferreira, A., et al. (2014) Morphometric Study (Macroscopic and Microscopic) of the Lower Abdominal Wall. Plastic and Reconstructive Surgery, 134, 1313-1322. https://doi.org/10.1097/PRS.0000000000000732

[28] Harley, O. and Pickford, M. (2013) CT Analysis of Fat Distribution Superficial and Deep to the Scarpa's Fascial Layer in the Mid and Lower Abdomen. Journal of Plastic, Reconstructive \& Aesthetic Surgery, 66, 525-530.

https://doi.org/10.1016/j.bjps.2012.12.003

[29] Avelar, J. (1989) Regional Distribution and Behavior of the Subcutaneous Tissue Concerning Selection and Indication for Liposuction. Aesthetic Plastic Surgery, 13, 155-165. https://doi.org/10.1007/BF01570212

[30] Vásconez, L. and De La Torre, J. (2006) Abdominoplasty. Plastic Surgery, 6, 87-118.

[31] Hunstad, J.P. and Repta, R. (2009) Anatomic Considerations in Abdominal Contouring. Atlas of Abdominoplasty, 1, 5-13. https://doi.org/10.1016/B978-1-4160-4080-4.00002-3

[32] Worseg, A.P., et al. (1997) Scarpa's Fascia Flap: Anatomic Studies and Clinical Application. Plastic and Reconstructive Surgery, 99, 1368-1380.

https://doi.org/10.1097/00006534-199705000-00026

[33] Felmerer, G., et al. (2002) The Lymphatic System of the Deep Inferior Epigastric Artery Perforator Flap: An Anatomical Study. British Journal of Plastic Surgery, 55, 335-339. https://doi.org/10.1054/bjps.2002.3830

[34] Nahas, F.X., Ferreira, L.M. and Ghelfond, C. (2007) Does Quilting Suture Prevent Seroma in Abdominoplasty? Plastic and Reconstructive Surgery, 119, 1060-1064. 
https://doi.org/10.1097/01.prs.0000242493.11655.68

[35] Stewart, K., et al. (2006) Complications of 278 Consecutive Abdominoplasties. Journal of Plastic, Reconstructive \& Aesthetic Surgery, 59, 1152-1155.

https://doi.org/10.1016/j.bjps.2005.12.060

[36] van Uchelen, J.H., Werker, P.M. and Kon, M. (2001) Complications of Abdominoplasty in 86 Patients. Plastic and Reconstructive Surgery, 107, 1869-1873. https://doi.org/10.1097/00006534-200106000-00037

[37] Neaman, K.C. and Hansen, J.E. (2007) Analysis of Complications from Abdominoplasty: A Review of 206 Cases at a University Hospital. Annals of Plastic Surgery, 58, 292-298. https://doi.org/10.1097/01.sap.0000239806.43438.54

[38] Khan, U.D. (2008) Risk of Seroma with Simultaneous Liposuction and Abdominoplasty and the Role of Progressive Tension Sutures. Aesthetic Plastic Surgery, 32, 93-99. https://doi.org/10.1007/s00266-007-9004-y

[39] Swanson, E. (2018) Evidence-Based Body Contouring Surgery and VTE Prevention. Springer, Berlin. https://doi.org/10.1007/978-3-319-71219-2

[40] Friedman, T., et al. (2015) Defining the Lymphatic System of the Anterior Abdominal Wall: An Anatomical Study. Plastic and Reconstructive Surgery, 135, 1027-1032. https://doi.org/10.1097/PRS.0000000000001136

[41] Janis, J.E., Khansa, L. and Khansa, I. (2016) Strategies for Postoperative Seroma Prevention: A Systematic Review. Plastic and Reconstructive Surgery, 138, 240-252. https://doi.org/10.1097/PRS.0000000000002245

[42] Chaouat, M., et al. (2000) Abdominal Dermolipectomies: Early Postoperative Complications and Long-Term Unfavorable Results. Plastic and Reconstructive Surgery, 106, 1614-1618. https://doi.org/10.1097/00006534-200012000-00029

[43] Manassa, E.H., Hertl, C.H. and Olbrisch, R.-R. (2003) Wound Healing Problems in Smokers and Nonsmokers after 132 Abdominoplasties. Plastic and Reconstructive Surgery, 111, 2082-2087. https://doi.org/10.1097/01.PRS.0000057144.62727.C8 